\title{
Bioactivity of Recombinant Humanized Monoclonal Antibody Against HER2 and Its Mechanism of Action on Ovarian Cancer
}

\section{Jinfeng Zeng}

Changhai Hospital

\section{Rui Zhang}

Changhai Hospital

Rui Guan ( $\nabla$ cngreen785@163.com )

Changhai Hospital https://orcid.org/0000-0002-5876-2695

\section{Research}

Keywords: HER2, recombinant humanized monoclonal antibody, transient gene expression system, HEK293F Cell line, antibody-dependent cellular cytotoxicity, NOD/SCID mouse model

Posted Date: October 1st, 2021

DOI: https://doi.org/10.21203/rs.3.rs-871731/v1

License: (c) (i) This work is licensed under a Creative Commons Attribution 4.0 International License. Read Full License 


\section{Abstract}

BACKGROUND: Human epidermal growth factor receptor 2 (HER2) protein is overexpressed on the surface of various epithelial ovarian cancer tissues, which mediates the proliferation, differentiation, metastasis and signal transduction of tumor cells and is a potential cancer therapeutic target.

METHODS: In this paper, the recombinant anti-HER2 humanized IgG1 monoclonal antibody (rhHer2-mAb) was expressed in HEK293F cells by constructing mammalian cell expression vector and optimizing transfection conditions. The antibody was purified by rProtein A affinity chromatography, and its mediated antibody dependent cytotoxicity (ADCC) was identified by lactate dehydrogenase(LDH) lactate dehydrogenase release assay. The anti-tumor activity of rhHer2-mAb was evaluated in NOD/SCID mice.

RESULTS: The expression of rhHer2-mAb in HEK293F cells was at the highest level (100.5 mg/L) when the ratios of DNA/PEI and light chain/heavy chain was $1: 4$ and $1: 2$, respectively. The $I_{50}$ on $A D C C$ of antibodies against SK-OV-3, OVCAR-3 and A-2780 cells were 12.36, 5.43 and $102.90 \mathrm{ng} / \mathrm{ml}$, respectively. Animal experiments in mice showed that rhHer2-mAb could effectively inhibit the growth of SK-OV-3 tumor at the dose of $10 \mathrm{mg} / \mathrm{kg}$.

CONCLUSIONS: The recombinant monoclonal antibody was obtained by transient gene expression technology and its bioactivity was studied in vitro and in vivo, providing a novel insight for the development and production of future biotechnology-based drugs using transient gene expression technology of HEK293F.

\section{Introduction}

Human epidermal growth factor receptor 2 (HER2), a transmembrane protein with tyrosine protein kinase activity and a molecular weight of 18500 , belongs to the epidermal growth factor receptor (EGFR) family. Overexpression of HER2 has been identified on the surface of various cancer cells such as colon, gastric, breast and ovarian cancers [1] Studies have demonstrated the essential role of HER2 in processes such as tumor cell proliferation, transformation and invasion and found that, beyond a prognostic indicator, HER2 is also an important therapeutic target for precision treatment [2]. Herceptin (trastuzumab) is a humanized monoclonal antibody targeting the extracellular domain of HER2 protein which works by down-regulating HER2 receptor expression and blocking ligand-mediated cell signal transduction. Currently, Herceptin has been widely used clinically to treat metastatic breast cancer with overexpression of HER2 protein. With the rapid development of targeted therapy, the safety and efficacy of trastuzumab in ovarian cancer have been extensively studied [3]. Ovarian cancer is a common gynecologic malignancy characterized by high mortality and the lack of effective modalities for early diagnosis and treating tumor metastasis. Therefore, it is of substantial importance to develop innovative treatment modalities and and novel therapies for ovarian cancer.

Transient gene expression (TGE), as a well-established technology for rapid protein production, can facilitate the rapid production of proteins with lower cost, shorter production period, and 
less dependence on stable transfection cell banks compared with traditional stable gene expression approaches. TGE can also enable rapid production of recombinant proteins in the development and manufacturing of biomacromolecule drugs, such as those used in pre-clinical compound screening, toxicology testing and early clinical drug production. In the application of TGE, factors with impacts on the yield of recombinant monoclonal antibody include host cell type, culture method, expression vector, molecular structure, and transfection method, as well as corresponding upstream and downstream processes [4]. Among others, human embryonic kidney cells (HEK293) are commonly used mammalian host cells in protein production, which can be suspended in large-scale culture. Protein products can be folded correctly and post-translationally modified, thus providing high transfection efficiency and yield[5]. Previous studies have tested the consistency of transient transfection technology and stable transfection expression products, and found that different batches of products produced by different technology platforms have consistency in affinity, secondary structure, thermal stability and glycosylation modification, which provided theoretical basis for the application of TGE in preclinical drug development [6]

In this study, the light and heavy chain expression vectors of rhHER2-mAb were built based on the HEK293F cell TGE system. Through optimizing the co-transfection system, rhHER2-mAb was purified and its affinity and antibody dependent cytotoxicity (ADCC) efficacy were further investigated. The in vivo and in vitro experiments in this study have laid the foundation for further study on the bioactivity of rhHER2-mAb and other novel antibodies.

\section{Materials}

\subsection{Materials}

Escherichia coli DH5a was prepared in our laboratory and the eukaryotic expression plasmid pCDNA3.4 was saved for this study. The plasmid was inserted into the constant regions of HER2 antibody light and heavy chain DNA coding fragments. SK-OV-3, OVCAR-3 and A-2780 cells were preserved in our laboratory and NOD/SCID mice were fed and kept in the laboratory.

\subsection{Devices and reagents}

DNA and protein gel electrophoresis devices and gel imaging analysis system were purchased from Shanghai Tianneng Technology, China. Reagents purchased and used in this study including: AKTA Start fast liquid system (GE Healthcare, USA); DNA polymerase and restriction endonucleases (Takara, Japan) ); homologous recombination kit (Nanjing Novazan Biotechnology, China); DNA gel recovery kit and nonendotoxic mass extraction kit (Axygen, USA); anti-human HER2 antibody (mouse anti-human monoclonal antibody, BD, USA), peroxidase AffiniPure donkey anti-Human IgG $(\mathrm{H}+\mathrm{L})$ antibody (Jackson, USA); trastuzumab (Herceptin, Roche, USA); ECL luminous chromogenic solution (Suzhou Xinsaimei Biotech, China); 25-kDa linear polyethyleneimine (PEl, Ployscience, USA); freestyle 293 medium, phenol red-free RPMI 1640 medium, McCoy's 5A Media, L-15 medium, DMEM medium, trypsin, fetal bovine serum and pancreatin (Gibco, USA and Jinuo Biomedical Technology, China); FBS (BI, Israel); rProtein affinity 
chromatography column packing (Yisheng Biotechnology, China). Sequencing and primer synthesis were completed with the help of Shanghai Sangon biotechnology, China.

\section{Methods And Results}

\subsection{Construction of the ihHER2-mAb expression vector}

The eukaryotic expression vector pCDNA3.4 was designed and built in this study utilizing the TGE system with mammalian cell HEK293F as the host cell. The variable region sequence obtained via screening of the laboratory phage library was spliced and cloned into the pCDNA3.4 plasmid to construct the rhHER2mAb expression vector (Fig1A,1B). The primers were designed according to the gene sequence shown

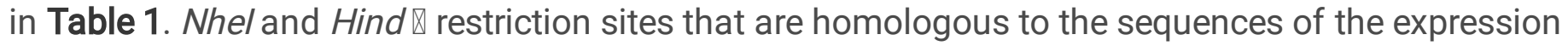
vector $p$ CDNA 3.4 were introduced at the 5 '-end of the primer. Overlap PCR was used to splice the gene fragments of light and heavy chains[7]. The light chain was obtained by overlap PCR using primers 1, 2, 3 , and 4 , while the heavy chain was obtained by primers $1,5,6$, and 7 . The $p$ CDNA3.4 plasmid double-

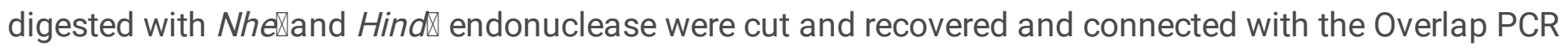
product using a homologous recombination kit. The homologous recombination product was then transformed into Escherichia coli DH5a. The positive clones are selected for culture and sent for sequencing, and mutation-free clones are selected for mass sampling of non-endotoxic plasmid. The result of $0.8 \%$ agarose gel electrophoresis showed that light and heavy chain sizes were $708 \mathrm{bp}$ and 1413 bp, respectively (Fig 1C). The final sequencing results confirmed that the expression vectors, namely pCDNA3.4-Anti-Her2 Light chain and pCDNA3.4-Anti-Her2 Heavy chain, were successfully constructed.

\subsection{Transient gene expression of rhHer2-mAb}

Conditions of TGE, such as DNA/PEI and heavy/light chain plasmid ratios, were optimized using HEK293F as the host cell and pCDNA3.4 as the eukaryotic expression plasmid[8].

\subsubsection{Optimization of DNA/PEI ratio}

The heavy and light chain plasmids were extracted using endotoxin-free plasmid extraction kit before filtration with the $0.22 \mathrm{~mm}$ filter; HEK293F cells were inoculated into Freestyle 293 medium and placed in a constant temperature shaker at $37^{\circ} \mathrm{C}, 5 \% \mathrm{CO}_{2}$, and $125 \mathrm{rpm}$. The cell density and viability were maintained at between $1-2 \times 10^{6}$ cells $/ \mathrm{ml}$ and above $95 \%$, respectively. Twenty-four hours before transfection, cells were centrifuged and resuspended in fresh medium before the expansion of cells to a density of $0.6 \times 10^{6}$ cells $/ \mathrm{ml}$. On the day of transfection, the cell concentration was measured before identification of cell types. Cell density was then adjusted to $1-1.2 \times 10^{6} \mathrm{cell} / \mathrm{ml}$ and $0.6 \mathrm{ml}$ of the cells was added to a 12-well plate. Based on the predetermined condition that $0.6 \mathrm{mg}$ of plasmid is needed to transfect every $10^{6}$ cells, the light/heavy chain plasmids were matched with a mass ratio of $1: 1$ and DNA and PEl were mixed with mass ratios of $1: 1,1: 2,1: 3,1: 4$ and 1:5, respectively and inoculated at room temperature for 25 min before being added to the cell suspension to continue culturing. The supernatant 
of the cell fermentation broth was collected by centrifugation $48 \mathrm{~h}$ later for subsequent cell identifications.

Meanwhile, the green fluorescent protein (GFP) and control plasmids were mixed with PEl using the same gradient ratio for transfection. After $48 \mathrm{~h}, 1 \times 10^{6}$ cells were collected from each group, centrifuged at 300 $\times g$ at $4^{\circ} \mathrm{C}$ for $5 \mathrm{~min}$, washed twice with $1 \mathrm{~mL}$ of $2 \%$ FBS-PBS buffer before resuspension to $100 \mathrm{ml}$, and passed through a flow cytometer. The transfection efficiency was detected by the FITC channel. The results showed that the transfection efficiencies of DNA/PEI ratio from 1:1 to $1: 5$ were $5.02 \%, 12.38 \%$, $35.99 \%, 90.22 \%$ and $92.34 \%$ (Fig $2 \mathrm{~A}$ ), respectively, suggesting that the transfection efficiency can exceed $90 \%$ when the DNA/PEI ratio is $1: 4$ and $1: 5$. In terms of cost, a DNA/PEI ratio of $1: 4$ was determined for subsequent transfection steps with a transfection efficiency of $90.22 \%$.

\subsubsection{Optimization of light/heavy chain to plasmid ratio}

Based on the aforementioned transfection method using different co-transfection ratios of light/heavy chain plasmid (Table 2), DNA and PEI were mixed at a mass ratio of 1:4 and inoculated at room temperature for 25 minutes before being added to the cell suspension for further culture. After $48 \mathrm{~h}$, the supernatant of the cell fluid was collected by centrifugation for subsequent analysis.

Western blotting was used to verify whether rhHER2-mAb was correctly assembled. Samples were isolated by $10 \%$ SDS-PAGE gel electrophoresis and transferred to PVDF membrane before incubation overnight at $4{ }^{\circ} \mathrm{C}$ with TBST containing $5 \%$ skimmed milk powder. The next day, the PVDF membrane was incubated with anti-human IgG $(\mathrm{H}+\mathrm{L})$ secondary antibody before rinsing with TBST for 3 times, each lasting for 5 min each time. Western blotting films were then developed. The impacts of DNA/PEI and light/heavy chain plasmid ratios on the transient expression of rhHER2-mAb were compared. Results showed that the protein yield is higher when DNA/PEI ratio was 1:4 and the light/heavy chain ratio was 2:1. Thus, the optimal ratios were adopted for subsequent large-scale expression of rhHer2-mAb.(Fig 2B,2C)

Therefore, based on the aforementioned transfection method, $250 \mathrm{ml} \mathrm{HEK293F} \mathrm{cells} \mathrm{were} \mathrm{transfected}$ with a light-to-heavy chain ratio of 2:1 and a DNA/PEI ratio of 1:4. When the cell viability is lower than $60 \%$, cell fermentation supernatant was collected by centrifugation at $800 \times \mathrm{g}$ for 20 min at $4^{\circ} \mathrm{C}$ before undergoing a second centrifugation at $8000 \mathrm{rpm}$ for $30 \mathrm{~min}$ and passing through a $0.22 \mathrm{~mm}$ filter for protein purification.

\subsection{Purification and identification of ihHER2-mAb r}

rProteinA has high specificity and affinity against the Fc segment at the stable region of the heavy chain, as well as has good versatility for antibody purification. All purification reagents are filtered with a $0.22 \mathrm{~mm}$ membrane, and cell fermentation broth supernatant was subsequently filtered through a $0.45 \mathrm{~mm}$ membrane. Specific processes of rProteinA affinity chromatography was as follows: The AKTA system was rinsed with $20 \%$ ethanol and ultrapure water, and the column was connected to Position 1 of 
the AKTA purifier. Binding buffer ( $\mathrm{pH} 7.2,20 \mathrm{mM}$ phosphate buffer, $150 \mathrm{mM} \mathrm{NaCl}$ ) was used to equilibrate the column and the sample was loaded at a speed of $1 \mathrm{ml} / \mathrm{min}$. After loading, the column was equilibrated again using binding buffer before non-specific impurities were removed by using the Wash buffer ( $\mathrm{pH}$ 5.0, $100 \mathrm{mM}$ citrate buffer). The protein was eluted with elution buffer $(100 \mathrm{mmol} / \mathrm{L}$ citric acid buffer, $\mathrm{pH}$ 3.0). The eluted sample was then adjusted to neutral $\mathrm{pH}$ with neutralization buffer $(1 \mathrm{~mol} / \mathrm{L}$ Tris- $\mathrm{HCl}$ buffer, $\mathrm{pH} 8.5$ ), and the column was eventually washed with $0.5 \mathrm{M} \mathrm{NaOH}$ and $20 \%$ ethanol. The obtained product antibody was dialyzed into PBS ( $\mathrm{pH} 7.2)$, filtered and sterilized, and stored in a $-80^{\circ} \mathrm{C}$ refrigerator.

Results of rProtein A affinity chromatography was shown in( Fig 3A)The upper column was cleaned with citric acid washing solution $(\mathrm{pH}$ 5.0) and replacement solution $(\mathrm{pH} 3.0)$ for purified products with no impurities eluted. rhHER2-mAb can be eluted in large scale using the replacement solution, presenting with a unified peak pattern. Finally, unintended products were eluted using $\mathrm{NaOH}$. SDS-PAGE gel electrophoresis results showed that the molecular weight of rhHER2 antibody was greater than $180 \mathrm{kDa}$, and the bands of interest for the reduced sample was clear. The heavy and light chains' molecular weights were measured to be 55 and $25 \mathrm{kDa}$, respectively (Fig 3B). The purified sample was dialyzed, concentrated and underwent protein quantification. The protein concentration was determined to be $7.3824 \mathrm{mg} / \mathrm{ml}$, with a yield of about $102.45 \mathrm{mg} / \mathrm{l}$.

\subsection{Affinity detection of rhHER2-mAb}

The affinity of rhHER2-mAb is determined by bio-layer interferometry (BLI) using OctetRed96 system with the following protocol: The streptomycin affinity (SA) sensor was pre-soaked in PBS buffer for 15 minutes and the program was set for antibody affinity detection[9]. The SA sensor was then infiltrated into the equilibrium solution ( $0.02 \%$ PBST containing $0.05 \% \mathrm{BSA}$ ) for $180 \mathrm{~s}$ as benchmark. The sensor was infiltrated into the solidification solution ( $5 \mathrm{mg} / \mathrm{ml}$ biotin-labeled Her2 protein) for $600 \mathrm{~s}$. After the signal was stable, the sensor was soaked in the fresh equilibrium solution for $180 \mathrm{~s}$, before being soaked to solutions with rhHER2-mAb concentrations of $50 \mathrm{nM}, 25 \mathrm{nM}, 12.5 \mathrm{nM}, 6.25 \mathrm{nM}$ and $3.125 \mathrm{nM}$, as well as PBS control, for $450 \mathrm{~s}$. After the signal was stable, the sensor was immersed in PBS and dissociated for $1800 \mathrm{~s}$. The above experiments were carried out at $30^{\circ} \mathrm{C}$ with a rotating speed of $1000 \mathrm{r} / \mathrm{min}$, and the PBS (pH 7.4) used in all steps was of the same batch. Data analysis was performed with the OctetRed96 analysis software. After subtracting the data of PBS control group from the that of the experimental group, the curve of binding and dissociation was depicted (Fig 4). The binding rate constant ( $\left.\mathrm{k}_{\text {on }}\right)$ was calculated as $2.69 \times 10^{5} 1 / \mathrm{Ms}$ and the dissociation rate constant ( $\left.k_{\text {off }}\right)$ was $9.43 \times 10^{-7} 1 / \mathrm{s}$. The equilibrium dissociation constant, i.e. the antibody affinity $\left(K_{D}\right)$ was $2.35 \times 10^{-13} M$ with an $R^{2}$ of 0.9538 .

\subsection{Biological activity of rhHER2-mAb}

\subsubsection{Selection of HER2-high-expression cell lines}


In terms of HER2 antigen over-expression on the cell surface, the SK-OV-3, OVCAR-3 and A2780 cell lines were selected as the cells of interest for the investigation of rhHER2-mAb bioactivity; Macoy'5A+10\% FBS, RPMI 1640+10\%FBS, and DMEM+10\%FBS complete medium were prepared respectively to culture SKOV-3, OVCAR-3 and A2780 cells in a $5 \% \mathrm{CO}_{2}$ incubator at $37^{\circ} \mathrm{C}$. Cell surface expression level of Her2 on our panel of cancer cells was tested using flow cytometry. $\nabla$ Fig 5A)

\subsubsection{Detection of rhHer2-mAb bioactivity}

In this study, the LDH cytotoxicity assay was used to detect the ADCC level mediated by rhHer2-mAb, in which lactate dehydrogenase (LDH) was quantitatively measured by CytoTox 96ß Cytotoxicity Detection Kit. According to manufacturer's instructions, the experimental and control groups, including effector cell spontaneous LDH release, target cell spontaneous LDH release, target cell maximum LDH release, volume correction control, and culture medium-only control groups, were set, with 3 replicates per group. Three strains of SK-OV-3, OVCAR-3 and A2780 cells respectively in growth phase were collected, centrifuged at 1000 rpm, resuspended in RPMI $1640+5 \%$ inactivated serum, sampled and counted, before subsequently plated in 96 well cell culture plates at a density of 8000 cells/well at $100 \mathrm{ml}$ per well. $\mathrm{A}_{490}$ was detected by a multifunctional microplate reader after sequentially adding LDH matrix liquid with stop solution according to manufacturer's instructions. Cytotoxicity ratios were calculated according to the formula, data were processed by GraphPad Prism 5, and the four-parameter analysis was adopted to fit a sigmoidal dose-dependent curve. The results showed that rhHER2-mAb exerted ADCC against all three cell lines at a PBMC ratio of 1:50, and the IC50 values of rhHER2-mAb against SK-OV-3, OVCAR-3, and A2780 cell lines were $6.06,96.49$, and $21.60 \mathrm{ng} / \mathrm{ml}$, respectively. . Fig 5B)

\section{6 rhHER2-mAb pharmacokinetics and anti-tumor animal experiments}

The experimental protocol was approved by the Institutional Animal Use Committee of Changhai Hospital. Blood samples were collected separately and diluted 2000 folds for quantitative determination by enzyme-linked immunosorbent assay (ELISA) standard procedures. Specifically, goat anti-human IgGkappa specific antibodies were incubated overnight on a 96-well high-affinity protein binding plate. rhHER2-mAB was captured using anti-human IgG-kappa antibody, and the signal was then amplified with goat anti-human FcHRP antibody. TMB solution was subsequently added to measure OD450 absorption. Finally, PK parameters were analyzed using a non-compartmental analysis model, and the half-life of rhHER2-mAb in vivo was found to be approximately 14 days (Fig 6A).

Antitumor efficacy evaluation of rhHER2-mAb mice were performed as follows: Fifteen female Balb/c mice (6-8 weeks old) were randomly divided into 3 groups, namely experimental rhHER2 group, Herceptin group and PBS group. Each mouse had subcutaneous implantation (on the right flank area) of 100ul of PBMC and SK-OV-3 cells, respectively, with an E/T ratio of 1:4. For each mouse, 5'10 ${ }^{6}$ SK-OV-3 cells cells were mixed with $2^{\prime} 10^{6}$ inactivated PBMCs in a volume of $100 \mu \mathrm{L}$. On the second day, mice in each group were injected in situ with $10 \mathrm{mg} / \mathrm{kg}$ rhHER2, Herceptin and PBS as blank controls, followed by weekly 
treatment via in situ injection of rhHER2-mAb at $10 \mathrm{mg} / \mathrm{kg}$ doses. The tumor size was measured with a vernier caliper every four days.

The anti-tumor bioactivity of rhHER2-mAb was evaluated on a xenograft NOD/SCID mouse model. Comparing the rhHER2-mAb, Herceptin and PBS groups after 60 days, results showed that rhHER2-mAb and Herceptin inhibited the growth of SK-OV-3 tumor. Further comparison between rhHER2-mAb and Herceptin was conducted and demonstrated that although both of them protected the mice from death at all, rhHER2-mAB was significantly more effective in inhibiting the growth of SK-OV-3 tumors after 60 days. Meanwhile, observations of subcutaneously denuded tumors found that mice in the PBS group had a more irregular tumor shape in addition to a larger size. (Fig 6B.6C.6D).

\section{Discussion}

Currently, monoclonal antibodies have become one of the mainstays in the biotherapeutic drug market for cancers. In the production of recombinant protein drugs, exogenous genes are introduced into host cells and triggers natural selection with screening markers. Exogenous genes are fully integrated into the host genome, and monoclonal cells with stable expression of desired target genes are finally selected [10]. The entire acquisition process of stable cells usually takes more than 6 months, while transient gene expression technology does not involve the integration of target genes into the host cell genome, thus avoiding the lengthy selection and screening process. However, the expression of the foreign gene is also lost with division of the host cell. Generally, the whole TGE process takes only a few weeks from the construction of the expression plasmid to acquisition of the target product, reducing the time and cost compared to stable transformation $[11,12]$

The transfection reagent used in TGE is mainly polyethyleneimine (PEI), which can adsorb to the cell membrane surface into cells by forming positively charged complexes with DNA. However, serum-free media often contains some components such as heparin and dextran sulfate to prevent cell aggregation, which would hinder the formation of positive complexes between PEI and DNA, thus affecting the transfection efficiency of PEI with the addition of extra steps like culture media exchanges in large-scale transient infection processes. Therefore, various medium that supports both high-density cell culture and PEI transformation have been developed, such as Freestyle medium from Invitrogen, which have greatly facilitated the scalability of TGE. Therefore, in this study, we further explored and selected Freestyle 293 medium and 293F cells, as well as $p$ CDNA3.4 expression vector, optimized the PEl-mediated transfection efficiency (1:4) and the co-transfection system with different plasmid ratios ( $\mathrm{Lc}$ : $\mathrm{Hc}=2: 1$ ), and finally achieved the goal of efficient target protein production.

Affinity of antibodies refers to the binding strength of the antigen-binding cluster of an antibody with the epitope of an antigen [8]. Methods for the detection of affinity mainly include enzyme-linked immunosorbent assay (ELISA), solid-phase radioimmunoassay, equilibrium dialysis, as well as Octect and Biacore assays. With bio-layer interferometry, the affinity of antibodies can be determined with the Fortebio Octet instrument and quantitative changes in the number of molecules on the sensor surface 
with related data on the concentration and kinetics can be obtained. In this study, the Octect assay was used to determine the $K_{D}$ value of rhHER2-mAb (2.35 pM), which is a monoclonal antibody with high affinity with comparable affinity and binding rate constant with marketed drugs. In a comparison of in vitro activity against different cells, rhHER2-mAb achieved a killing effect against SK-OV-3 at a low concentration, with an $\mathrm{IC}_{50}$ of $6.06 \mathrm{ng} / \mathrm{ml}$.

Xenograft NOD/SCID mouse models can be used to evaluate the anti-tumor bioactivity of antibodies[13]. Although these mice have severely combined immunodeficiency without functional $T$ and $B$ lymphocytes, the appearance and body weight of SCID mice are not different from normal mice despite severely atrophied thymus and peripheral lymphatic tissues. This mouse model is having more broad-spectrum usage in cancer immunotherapy. Therefore, this study evaluated the anti-tumor bioactivity of rhHER2mAb through the NOD/SCID mouse model and found that the purified rhHER2-mAb after transient expression could effectively inhibit the growth of tumors compared to the control group and achieve more effective killing of tumors in an ovarian cancer mouse model compared to Herceptin.

\section{Conclusion}

In this study, the ADCC effect mediated by rhHER2-mAb was investigated and a mechanism of action through the effective binding of rhHER2-mAb to the HER2 antigen on the cell surface was identified . When the antibody binds to the antigen on the target cell, the Fc region of the antibody undergoes conformational changes and subsequently binds to Fc receptors on effector $T$ cells, thereby mediating an ADCC effect $[14,15]$. In this study, the rh-HER2 humanized recombinant antibody protein was produced with HER2 as the target, and its ADCC effect on different cell lines was studied. Verification in a mouse model have also demonstrated that the antibody can effectively inhibit HER2 high-expressing cell lines in vivo, which, in a nutshell, provides technical support for the future development of HER2-targeted drugs and have provided evidence for humanized monoclonal antibody development using TGE in HEK293F.

\section{Abbreviations}

HER2:Human epidermal growth factor receptor 2 ;ADCC:Antibody dependent cytotoxicity ;LDH:Lactate dehydrogenase;

EGFR: Epidermal growth factor receptor ;TGE:Transient gene expression ; HEK293 :human embryonic kidney cells; GFP:Green fluorescent protein ; BLI: Bio-layer interferometry ; PEl: Polyethyleneimine ;ELISA:Enzyme-linked immunosorbent assay ;

\section{Declarations}

Acknowledgements: Not applicable.

\section{Author contributions}


Jinfeng Zeng $\square$ Hui Zhang contributed to the study design,completed the experiment,performed statistical analysis,interpreted the study results, and drafted the manuscript.

Rui Guan contributed to the study design, planned statistical analysis,oversaw the conduct of the statistical analysis,revised and approved the manuscript .

All authors had access to all of the data and take full responsibility for the integrity of the data,the accuracy of the data analysis,the finished manuscriptl and the decision to submit the manuscript.

Funding: Not applicable.

\section{Availability of data and materials:}

The datasets used and/or analyzed during the current study are available from the corresponding author on reasonable request.

\section{Ethics approval and consent to participate}

In this paper,all animal studies and experimental procedures were approved by Ethical Committee of changhai Hospital and conducted in accordance with the ethical standards.

\section{Competing interests}

The authors declare that they have no competing interests.

\section{Author details}

First Author: Jinfeng Zeng Department of Obstetrics and Gynecology, Changhai Hospital, Naval Military Medical University,Shanghai, China; E-mail:fengcoffee323@sohu.com; Tel:8615021069948

Co-first Author: Hui Zhang Department of Obstetrics and Gynecology, Changhai Hospital, Naval Military Medical University,Shanghai, China; E-mail:1472268517@qq.com; Tel:8613817344986

Corresponding Author:Rui Guan Department of Obstetrics and Gynecology, Changhai Hospital, Second

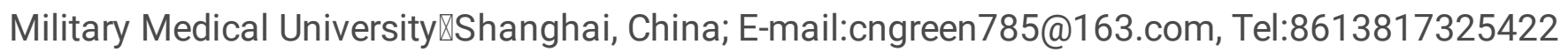

\section{References}

1. Tandon AK, Clark GM, Chamness GC, Ullrich A, McGuire WL. HER-2/neu oncogene protein and prognosis in breast cancer. J Clin Oncol. 1989;7:1120-8.

2. Slamon DJ, Clark GM, Wong SG, Levin WJ, Ullrich A, McGuire WL. Human breast cancer: correlation of relapse and survival with amplification of the HER-2/neu oncogene.Science. 1987;235:177-182.

3. Cameron D, Piccart-Gebhart MJ, et al. Herceptin Adjuvant (HERA) Trial Study Team. 11 years' followup of trastuzumab after adjuvant chemotherapy in HER2-positive early breast cancer: final analysis 
of the HERceptin Adjuvant (HERA) trial. Lancet. 2017 Mar;25(10075):1195-205. 389(.

4. Gutiérrez-Granados S, Cervera L, Kamen AA, Gòdia F. Advancements in mammalian cell transient gene expression (TGE) technology for accelerated production of biologics. Crit Rev Biotechnol. 2018 Sep;38(6):918-40.

5. König Jens, H Michael, van den. Heuvel Joop. Validation of the Production of Antibodies in Different Formats in the HEK 293 Transient Gene Expression System.. 2021, 2247:59-76.

6. DING K, HAN L, ZONG H, et al. Production process reproducibility and product quality consistency of transient gene expression in HEK293 cells with anti-PD1 antibody as the model protein [J]. Appl Microbiol Biotechnol. 2017;101(5):1889-98.

7. HECKMAN K L PEASELR. Gene splicing and mutagenesis by PCR-driven overlap extension [J]. Nat Protoc. 2007;2(4):924-32.

8. Rudnick SI, Lou J, Shaller CC, etc. Influence of affinity and antigen internalization on theuptake and penetration of Anti-HER2 antibodies in solid tumors[J]. Cancer Res., 2011, 71(6): 2250-2259.

9. Concepcion J, Witte K, Wartchow C, et al. Label-free detection of biomolecular interactions using BioLayer interferometry for kinetic characterization. Comb Chem High Throughput Screen. 2009 Sep;12(8):791-800.

10. Burmester GR, Panaccione R, Gordon KB, et al. Adalimumab: Long-term safety in 23458 patients from global clinical trials in rheumatoid arthritis, juvenile idiopathic arthritis, ankylosing spondylitis, psoriatic arthritis, psoriasis and Crohn's disease. Ann Rheum Dis. 2013;72:517-24.

11. ZHU J. Update on Production of Recombinant Therapeutic Protein: Transient Gene Expression [M]. Shropshire: Smithers Papra; 2013.

12. ZHU J. Mammalian cell protein expression for biopharmaceutical production [J]. Biotechnol Adv. 2012;30(5):1158-70.

13. Ito M, Hiramatsu $H$, Kobayashi K. etc. NOD/SCID/gamma(c)(null) mouse: an excellent recipient mouse model for engraftment of human cells. Blood. 2002 Nov 1;100(9):3175-82.

14. Pereira NA, Chan KF, Lin PC, Song Z. The "less-is-more" in therapeutic antibodies: Afucosylated anticancer antibodies with enhanced antibody-dependent cellular cytotoxicity. MAbs. 2018 Jul;10(5):693-711.

15. Laurie Lajoie L, Lajoie N, Congy-Jolivet, et al. Gradual Increase of FcyRIIla/CD16a Expression and Shift toward IFN-y Secretion during Differentiation of CD56dim Natural Killer Cells. 2017, 8: 15561567.

\section{Tables}

Table 1. Primers of rhHer2-mAb Light Chain and Heavy Chain 


\begin{tabular}{|c|c|c|}
\hline Primer & & Sequence $₫ 5^{\prime}-3^{\prime} \rrbracket$ \\
\hline 1 & Forward & CGAACCCTTGCTAGCACCATGGGTTGGAGCCTCATC \\
\hline 2 & Reverse & CGCTGGGCCCCTTCGTCTTGATCTCCACCTTGGT \\
\hline 3 & Forward & ACCAAGGTGGAGATCAAGACGAAGGGGCCCAGCG \\
\hline 4 & Reverse & GATCGAACCCTT $\underline{A A G C T T T C A C C C G G G G C T C A G G C T C ~}$ \\
\hline 5 & Reverse & CGCTGGGCCCCTTCGTGCTGCTCACGGTGACGAGG \\
\hline 6 & Forward & СCTCGTCACCGTGAGCAGCACGAAGGGGCCCAGCG \\
\hline 7 & Reverse & GATCGAACCCTT $\underline{A A G C T T T C A G C A C T C G C C C C G G T T G ~}$ \\
\hline
\end{tabular}

Table 2. Optimization of the co-transfection ratio of rhHER2-mAb expression vectors

Co-transfection ratio of light/heavy chain plasmid

\begin{tabular}{lllllllllll} 
Lc \% & 83.4 & 80 & 75 & 60 & 66.6 & 50 & 20 & 40 & 25 & 33.3 \\
\hline Hc \% & 16.6 & 20 & 25 & 40 & 33.3 & 50 & 80 & 60 & 75 & 66.6
\end{tabular}

Figures

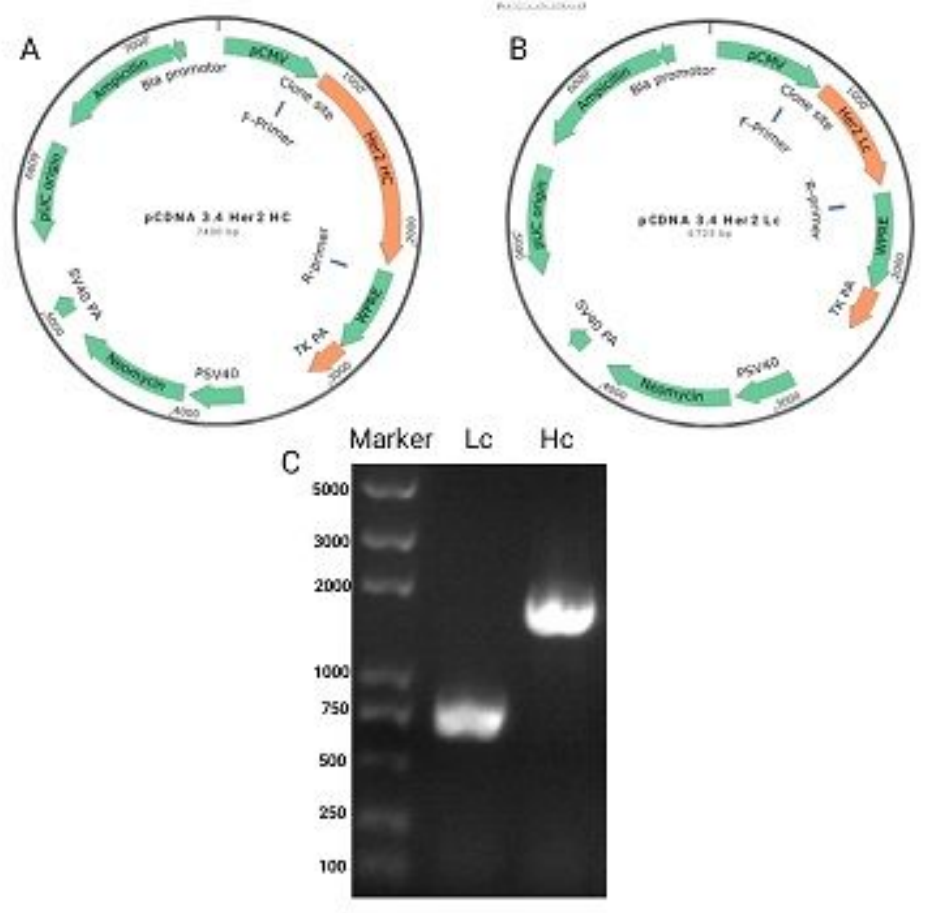

Figure 1 
Constructions of rhHer2-mAb expression vectors A. Expression vector map of rhHer2-mAb light chain, B. Expression vector map of rhHer2-mAb heavy chain; C. PCR result of rhHer2-mAb expression vector
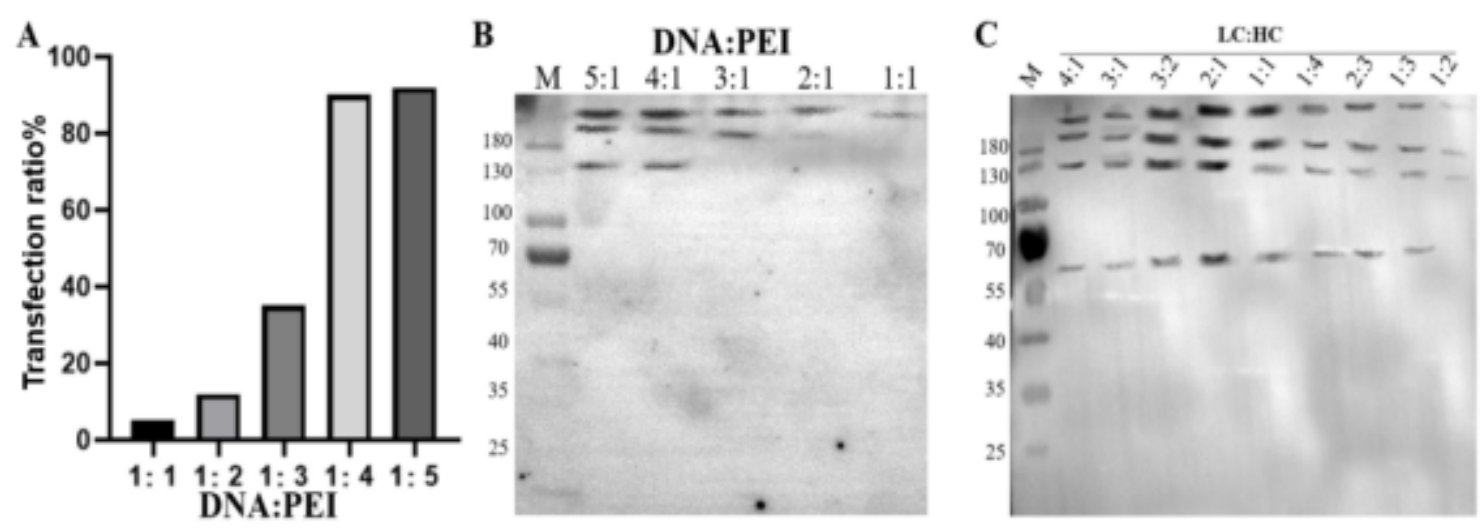

Figure 2

Optimization of transient gene expression conditions for rhHER2-mAb A. Transfection efficiency at different DNA/PEI ratios; $B$, Antibody expression at different DNA/PEI ratios was determined by Western blotting; C, Antibody (non-reduced) expression at different LC/HC plasmid ratios was assessed by Western blotting 


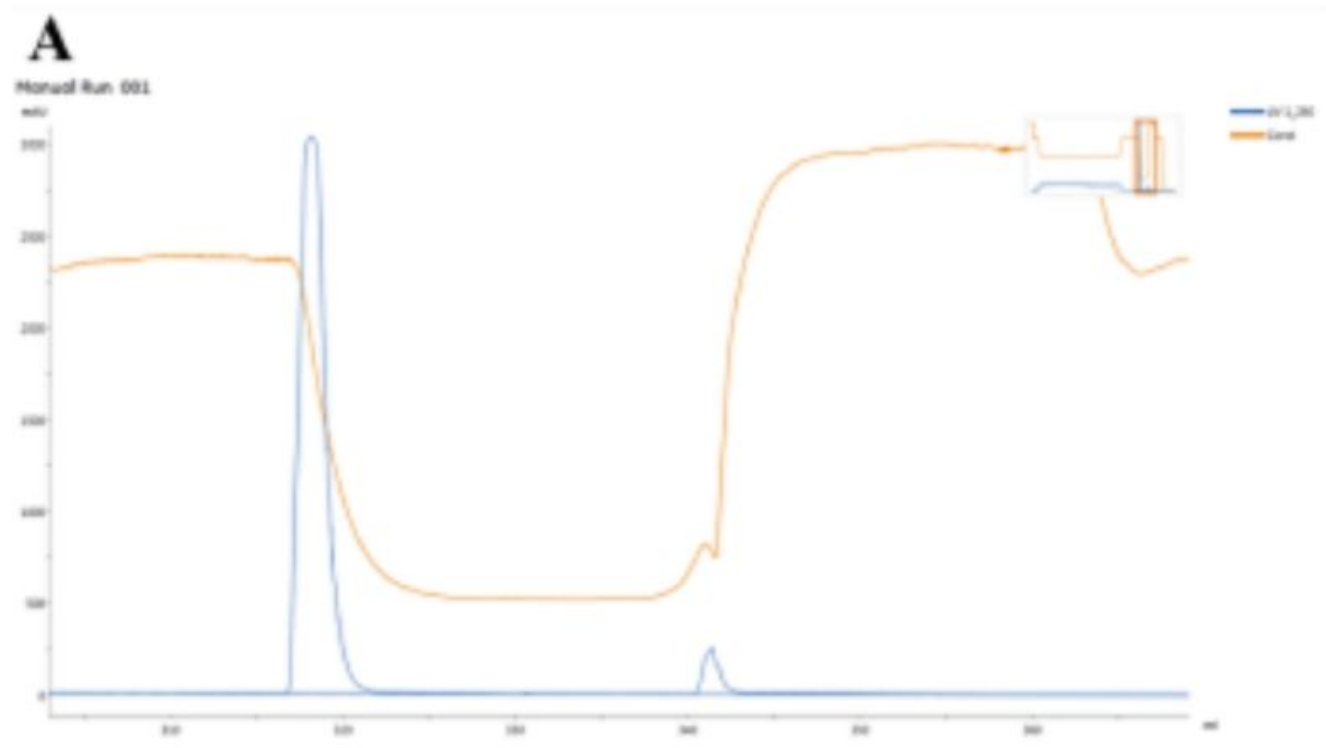

B

\section{$\begin{array}{lllllllllllll}M & 1 & 2 & 3 & 4 & \text { M } & 5 & 6 & 7 & 8\end{array}$}

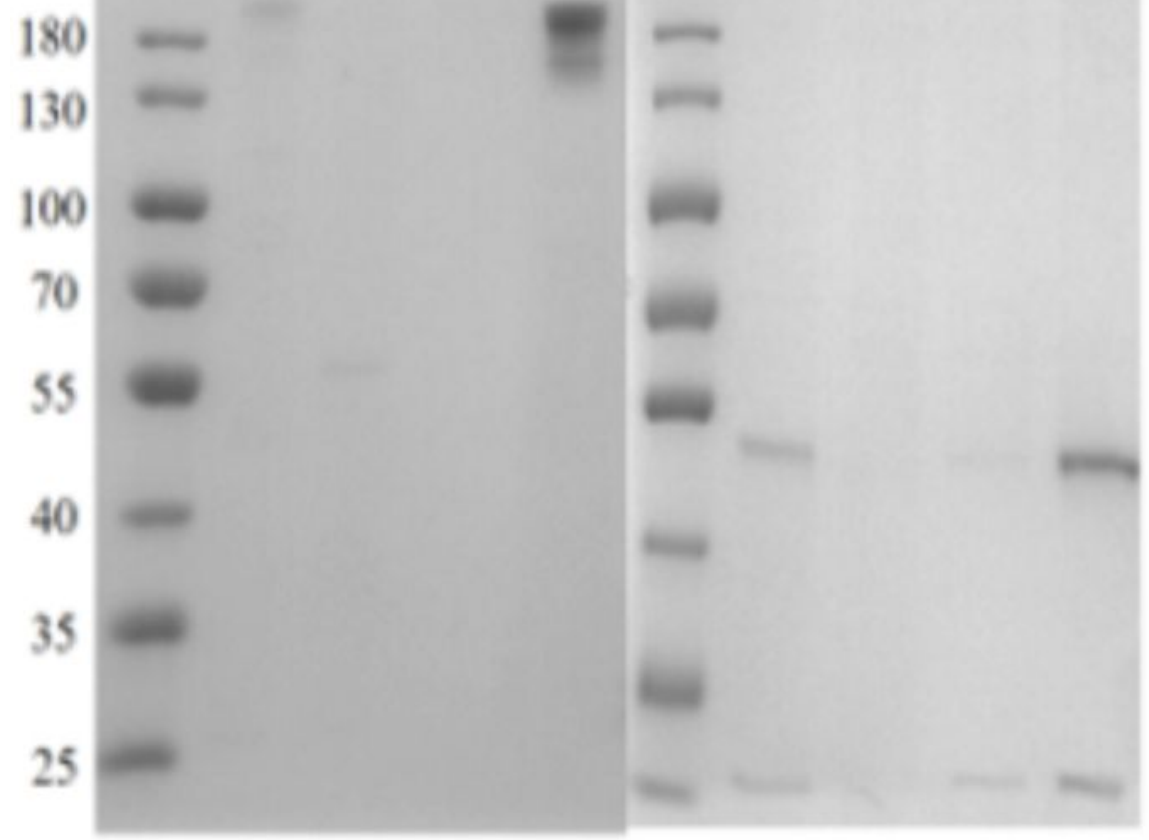

\section{Figure 3}

Purification of rhHer2-mAb by protein A affinity chromatography A: Peak pattern of eluted rhHER2-mAb in rProtein A affinity chromatography, B: SDS-Page purification results of rhHER2-mAb M-protein marker; 1cell supernatant before loading (non-reduced); 2-purification flow-through (non-reduced); 3, 4-eluted rhHER2-mAb sample (non-reduced); 5-cell supernatant before loading (reduced); 6-purification flowthrough (reduced); 7, 8-eluted rhHER2-mAb sample (reduced) 


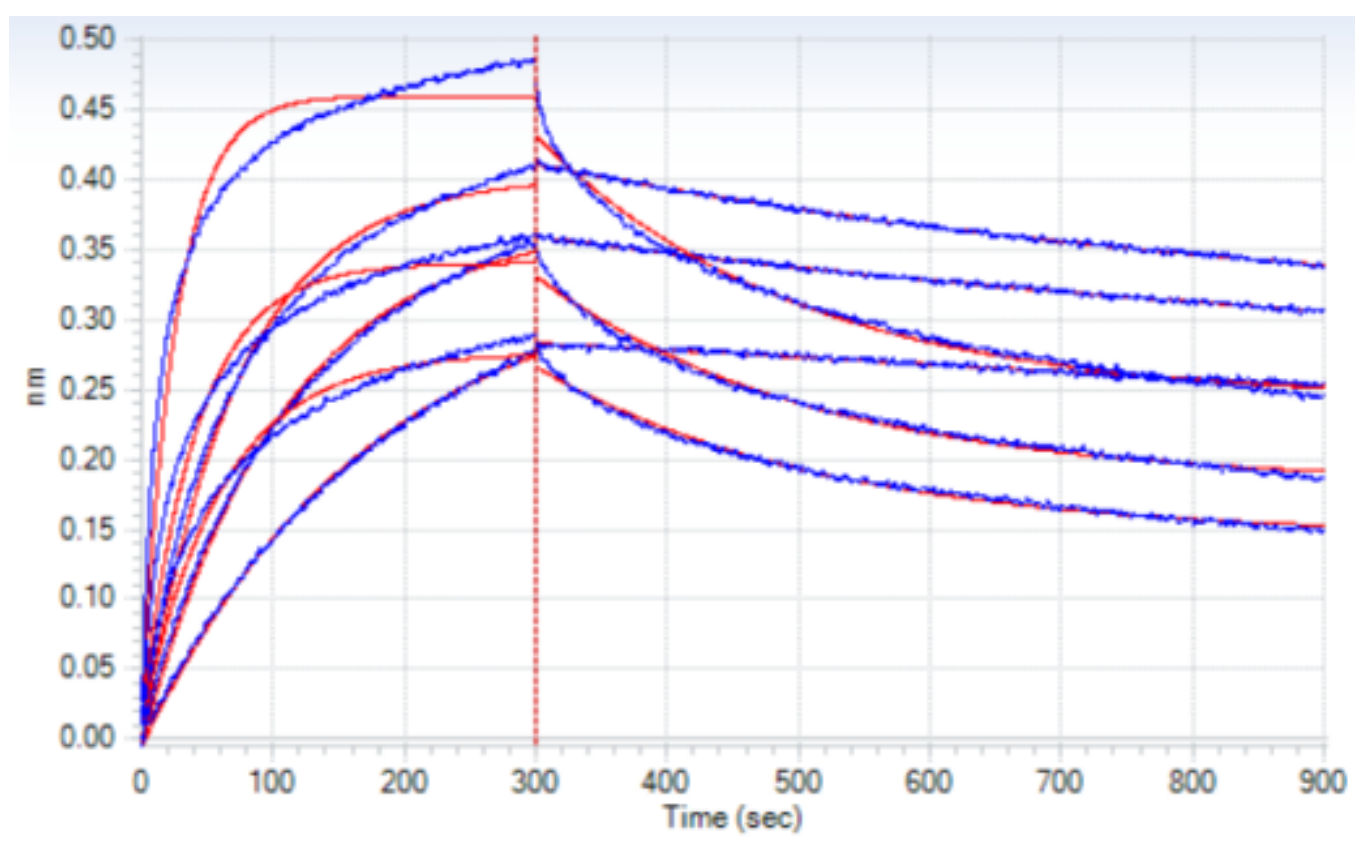

Figure 4

Affinity analysis of rhHER2-mAb by BLI

A

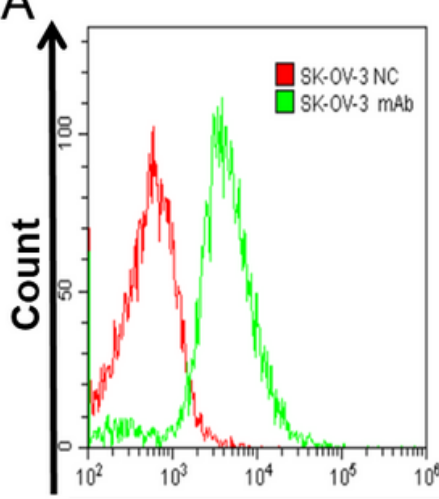

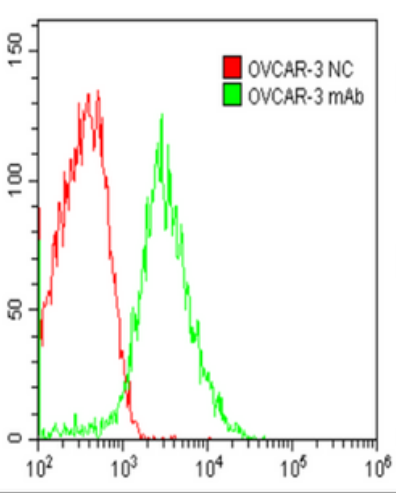

FITC
B

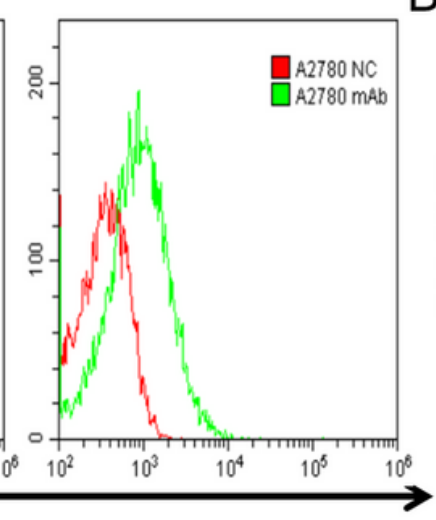

$\longrightarrow$

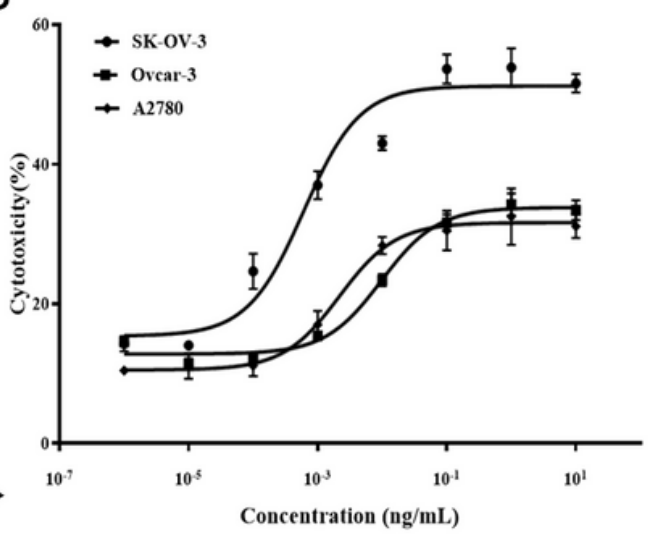

Figure 5

HER2-high-expression cell lines selection and ADCC assay of rhHER2-mAb by LDH releasing assay 

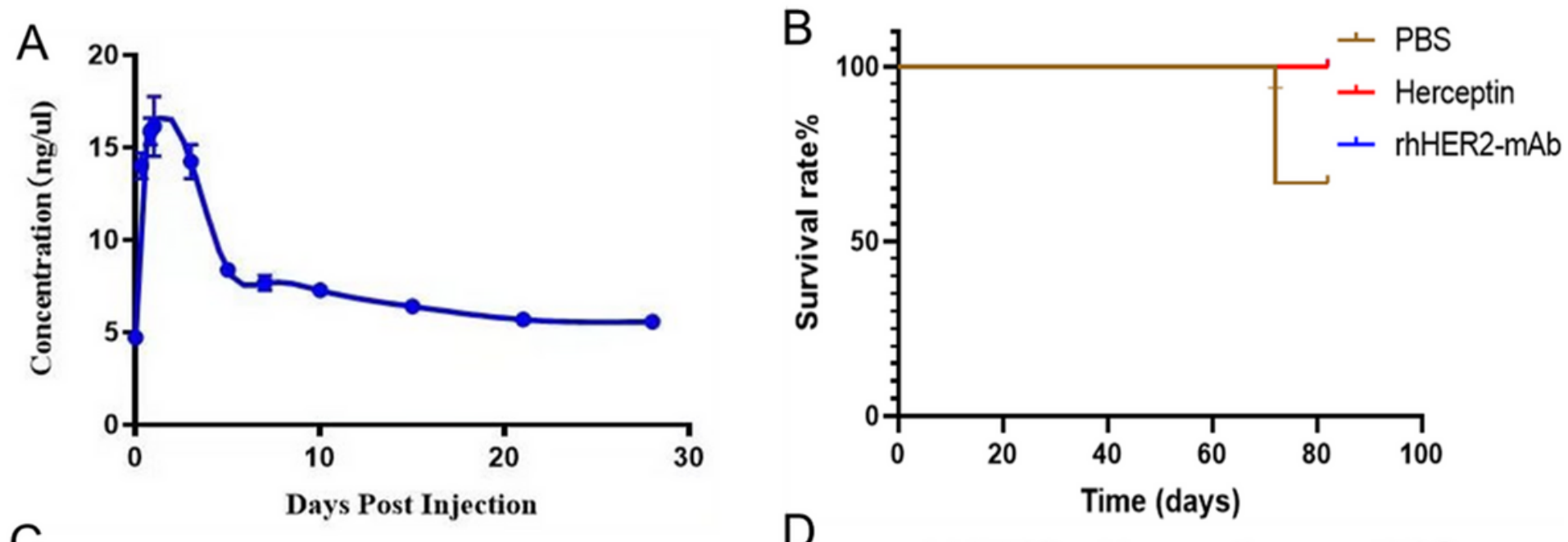

C

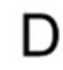

D rhHER2 Herceptin PBS
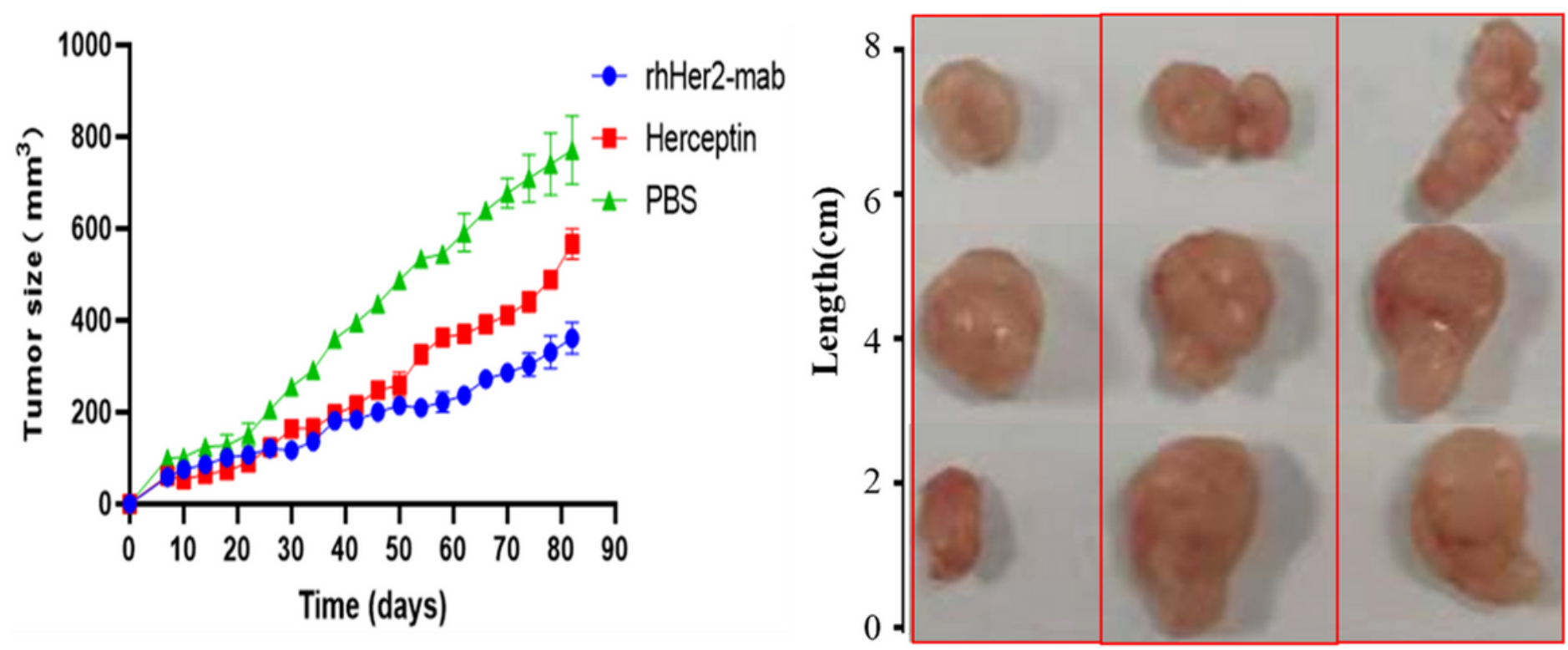

Figure 6

rhHER2-mAb treated mice in animal experiments $A$ : In vivo pharmacokinetics of rhHER2-mAb in mice; $B$ : Kaplan-Meier curve of rhHER2-mAb treated animals; C: Tumor size of rhHER2-mAb treated mice; D: subcutaneous tumors of mice in different groups 